\title{
Pitfalls in measuring the endocannabinoid 2-arachidonoyl glycerol in biological samples
}

\author{
Michael Vogeser ${ }^{1, *}$ and Gustav Schelling ${ }^{2}$ \\ ${ }^{1}$ Institute of Clinical Chemistry, Hospital of the \\ University of Munich, Munich, Germany \\ ${ }^{2}$ Department of Anesthesiology, Hospital of the \\ University of Munich, Munich, Germany
}

\begin{abstract}
Background: The endocannabinoid 2-arachidonoyl glycerol (2-AG) undergoes spontaneous isomerization to biologically inactive 1-AG. This effect has not been adequately addressed in previous studies that reported 2-AG concentrations in biological samples.

Methods: Liquid chromatography tandem-mass spectrometry (LC-MS/MS) was used for 1-AG and 2AG analyses.

Results: Identical collision-induced disintegration spectra were found for 1-AG and 2-AG. For specific detection of both compounds, which share a common mass transition, baseline chromatographic separation is mandatory, even when applying MS/MS technology with its generally high detection specificity. When using standard chromatographic conditions with the very short run times typically used in LC-MS/ MS methods, co-elution of 2-AG with 1-AG, which is present in human serum, causes false 2-AG results.

Conclusions: Our data highlight that the analytical specificity of MS/MS can be limited by interference from isobaric isomers with identical disintegration patterns. The specificity of this technology must be carefully evaluated for each individual application. Clin Chem Lab Med 2007;45:1023-5.
\end{abstract}

Keywords: 1-arachidonoyl glycerol (1-AG); 2-arachidonoyl glycerol (2-AG); endocannabinoids; interference; isomers; liquid chromatography tandem-mass spectrometry (LC-MS/MS).

During recent years it has become evident that the endocannabinoid system is part of fundamental regulatory mechanisms involved in numerous physiological processes (1). While endocannabinoid $\mathrm{CB}_{1}$ receptors are abundant in the central nervous system, $\mathrm{CB}_{2}$ receptors have been found primarily on peripheral immune and endothelial cells. Anandamide and 2-arachidonoyl glycerol (2-AG), both lipid mediators

*Corresponding author: Michael Vogeser, MD, Institute of Clinical Chemistry, Hospital of the University of Munich,

Marchioninistraße 15, 81377 Munich, Germany

Phone: +49-89-70953221, Fax: +49-89-70953240

E-mail: michael.vogeser@med.uni-muenchen.de derived from membrane phospholipids or triglycerides, are the most important ligands of these receptors.

Most of our knowledge of the functional principles of the endocannabinoid systems is based on experiments in which endocannabinoids or analogues were administered to animals or in which the effects of blocking the enzymatic inactivation of endocannabinoids were investigated. Apparent concentrations of 2-AG in various sample materials have been reported in a number of investigations that used liquid chromatography (LC)-single-stage mass spectrometry (MS) technology. However, an essential problem regarding the measurement of 2-AG in biological samples is that it undergoes rapid non-enzymatic isomerization to 1-arachidonoyl glycerol (1-AG), which is biologically inactive (2). It appears that this issue has not been adequately addressed in the literature. This problem has either been ignored (3-6), or 1-AG and 2-AG have been separated chromatographically, but the concentrations of both compounds have been added and a summed value has been reported (7-10). This approach is also inappropriate, since 1-AG is biologically inactive.

We investigated whether the technique of liquid chromatography tandem-mass spectrometry (LC-MS/ MS), which is substantially more specific compared to LC-MS with a single-quadrupole mass analyzer, would allow specific quantification of 2-AG in serum within short analytical run times.

In a first step, we recorded collision-induced disintegration mass spectra from solutions of 2-AG and 1-AG (Cayman Chemicals, Ann Arbor, MI, USA) by direct infusion. These spectra were undistinguishable, with a predominant fragment ion of $287 \mathrm{~m} / \mathrm{z}$. Consequently, it was evident that baseline chromatographic separation of the two compounds is mandatory to achieve specific detection, even when applying the highly specific LC-MS/MS technology.

We modified our previously described on-line LCMS/MS method for the quantification of anandamide (11) using a longer column and a reduced proportion of organic solvent in the analytical mobile phase $(85 \%$ methanol instead of $90 \%$ ). The mass transition $379>287$ was used for the detection of both 1-AG and 2-AG. Using these conditions, a single peak at a retention time of approximately $8.0 \mathrm{~min}$ was observed on injection of a pure solution of 1-AG. Injection of the 2-AG standard solution showed a main peak at approximately $7.4 \mathrm{~min}$ and a minor peak at approximately $8.0 \mathrm{~min}$. This demonstrated contamination of the standard solution with 1-AG. Injection of precipi- 


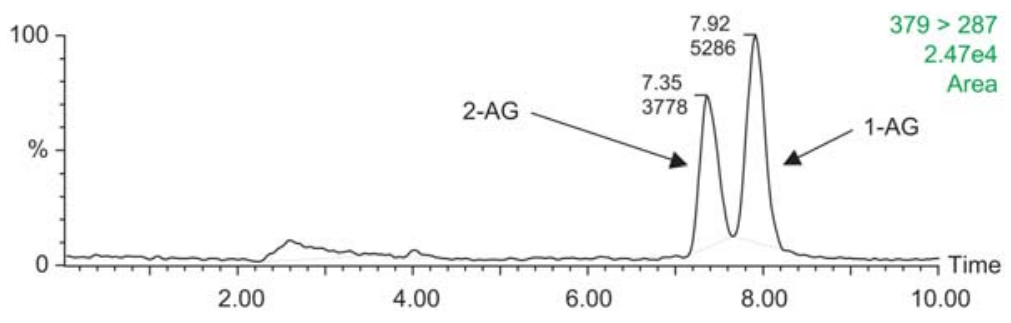

Figure 1 Representative LC-MS/MS chromatogram of a precipitated serum sample.

tated serum samples showed intense peaks at the retention times for both 2-AG and 1-AG, indicating the presence of relevant amounts of $1-A G$ in human serum.

When applying the chromatographic conditions originally used for anandamide measurement, a single peak for the (non-specific) mass transition $379>287$ was observed due to co-elution of 1-AG and 2-AG. Consequently, such an assay with a short chromatographic run time would be subject to isomer interference if used for 2-AG measurement.

To study the impact of delayed processing of serum, a freshly obtained serum sample was precipitated immediately and after $1 \mathrm{~h}$ of incubation at room temperature for comparison. The peak area ratio between the signals for 2-AG and 1-AG was substantially different (0.7 vs. 0.2 after incubation). This demonstrated that isomerization of 2-AG to 1-AG also occurs in serum (Figure 1).

In general, our results highlight that isomers of an individual target analyte may represent an important source of analytical error, even when applying LC-MS/ MS with its potentially high analytical specificity. Similar interferences can arise from in-source fragmentation of conjugate metabolites (12). Potential isomer and metabolite interference has already been reported for LC-MS/MS measurement of methylmalonic acid (13), 25-hydroxyvitamin $D_{3}(14)$, and cyclosporin A (15). Consequently, for specific detection of some target analytes, rather long chromatographic run times with appropriate separation efficacy may be mandatory, even for LC-MS/MS.

We conclude from our observations that a shortcoming of the literature on 2-AG concentrations in biological samples is that assay specificity towards 1-AG has not been addressed appropriately. Thus, the results of previous studies are questionable in the absence of statements on the method specificity with respect to 1-AG or if summed values for active 2-AG and inactive 1-AG have been reported. The development of reliable MS methods for quantification of 2AG in the future has to consider the instability of the target analyte in both standard materials and human serum samples. Therefore, the impact of preanalytical handling conditions on apparent 2-AG serum concentrations must be addressed carefully, as has been done for anandamide (11). Efforts to "trap" 2-AG in samples by addition of compounds interfering with its isomerization to 1-AG might be undertaken to allow accurate 2-AG measurement. Finally, calibration of specific 2-AG assays has to be corrected for the degree of analyte decay in the standard materials used.

\section{References}

1. Pagotto U, Marsicano G, Cota D, Lutz B, Pasquali R. The emerging role of the endocannabinoid system in endocrine regulation and energy balance. Endocr Rev 2006; 27:73-100.

2. van der Stelt M, van Kuik JA, Bari M, van Zadelhoff G, Leeflang BR, Veldink GA, et al. Oxygenated metabolites of anandamide and 2-arachidonoylglycerol: conformational analysis and interaction with cannabinoid receptors, membrane transporter, and fatty acid amide hydrolase. J Med Chem 2002;45:3709-20.

3. Marsicano G, Wotjak CT, Azad SC, Bisogno T, Rammes G, Cascio MG, et al. The endogenous cannabinoid system controls extinction of aversive memories. Nature 2002;418:530-4.

4. Hohmann AG, Suplita RL, Bolton NM, Neely MH, Fegley $D$, Mangieri R, et al. An endocannabinoid mechanism for stress-induced analgesia. Nature 2005;435:1108-12.

5. Blüher M, Engeli S, Klöting N, Berndt J, Fasshauer M, Batkai $S$, et al. Dysregulation of the peripheral and adipose tissue endocannabinoid system in human abdominal obesity. Diabetes 2006;55:3053-60.

6. Matias I, Gonthier MP, Orlando P, Martiadis V, De Petrocellis $L$, Cervino $C$, et al. Regulation, function, and dysregulation of endocannabinoids in models of adipose and beta-pancreatic cells and in obesity and hyperglycemia. J Clin Endocrinol Metab 2006;91:3171-80.

7. Ferrer B, Asbrock N, Kathuria S, Piomelli D, Giuffrida A. Effects of levodopa on endocannabinoid levels in rat basal ganglia: implications for the treatment of levodopa-induced dyskinesias. Eur J Neurosci 2003;18:1607-14.

8. Engeli S, Bohnke J, Feldpausch M, Gorzelniak K, Janke $\mathrm{J}$, Batkai $\mathrm{S}$, et al. Activation of the peripheral endocannabinoid system in human obesity. Diabetes 2005;54: 2838-43.

9. Van Sickle MD, Duncan M, Kingsley PJ, Mouihate A, Urbani $P$, Mackie $K$, et al. Identification and functional characterization of brainstem cannabinoid CB2 receptors. Science 2005;310:329-32.

10. Suplita RL II, Gutierrez T, Fegley D, Piomelli D, Hohmann AG. Endocannabinoids at the spinal level regulate, but do not mediate, nonopioid stress-induced analgesia. Neuropharmacology 2006;50:372-9.

11. Vogeser M, Hauer D, Christina Azad S, Huber E, Storr M, Schelling G. Release of anandamide from blood cells. Clin Chem Lab Med 2006;44:488-91.

12. Vogeser M, Zachoval R, Spohrer U, Jacob K. Potential lack of specificity using electrospray tandem-mass spectrometry for the analysis of mycophenolic acid in serum. Ther Drug Monit 2001;23:722-4.

13. Magera MJ, Helgeson JK, Matern D, Rinaldo P. Methylmalonic acid measured in plasma and urine by stable- 
isotope dilution and electrospray tandem mass spectrometry. Clin Chem 2000;46:1804-10.

14. Singh RJ, Taylor RL, Reddy GS, Grebe SK. C-3 epimers can account for a significant proportion of total circulating 25-hydroxyvitamin D in infants, complicating accurate measurement and interpretation of vitamin $D$ status. J Clin Endocrinol Metab 2006;91:3055-61.
15. Vogeser M, Spohrer U. Pitfall in the high-throughput quantification of whole blood cyclosporin $\mathrm{A}$ using liquid chromatography-tandem mass spectrometry. Clin Chem Lab Med 2005;43:400-2.

Received February 1, 2007, accepted May 3, 2007 